\title{
La dévolution dans les activités physiques sportives et artistiques non scolaires
}

Maël Le Paven, Sophie Roesslé, Emmanuelle Roncin, Monique Loquet et Yvon Léziart

\section{(2) OpenEdition}

\section{Journals}

Édition électronique

URL : https://journals.openedition.org/educationdidactique/189

DOI : 10.4000/educationdidactique.189

ISBN : 978-2-7535-1616-8

ISSN : $2111-4838$

\section{Éditeur}

Presses universitaires de Rennes

Édition imprimée

Date de publication : 1 décembre 2007

Pagination : $9-29$

ISBN : 978-2-7535-0597-1

ISSN : 1956-3485

\section{Référence électronique}

Maël Le Paven, Sophie Roesslé, Emmanuelle Roncin, Monique Loquet et Yvon Léziart, « La dévolution dans les activités physiques sportives et artistiques non scolaires », Éducation et didactique [En ligne], 1-3 | Décembre 2007, mis en ligne le 01 décembre 2009, consulté le 16 août 2022. URL : http:// journals.openedition.org/educationdidactique/189 ; DOI : https://doi.org/10.4000/ educationdidactique.189 


\title{
LA DÉVOLUTION DANS LES ACTIVITÉS PHYSIQUES SPORTIVES ET ARTISTIQUES NON SCOLAIRES
}

\author{
Maël Le Paven, Sophie Roesslé, Emmanuelle Roncin, \\ Monique Loquet \& Yvon Léziart \\ CREAD, IUFM de Bretagne, Université Rennes 2
}

\begin{abstract}
Résumé : Cet article a pour objectif d'identifier différentes manifestations de la dévolution, à travers l'étude fine de trois situations d'activités physiques sportives et artistiques (APSA), observées dans des institutions non scolaires de transmission (entraînement sportif, éducation spécialisée des handicapés, éducation de la petite enfance),. Nous examinons dans quelle mesure et sous quelle(s) forme(s) l'acte dévolutif est descriptible, au sein de ces institutions, selon deux niveaux de généricité : le premier permettant de comparer toute pratique d'intervention, scolaire et non scolaire; le second permettant d'ébaucher un modèle commun aux interventions non scolaires. Cette étude, en cherchant à élucider la nature dévolutive des relations didactiques non scolaires, décrit précisément les caractéristiques du milieu sur le fonds duquel ces relations s'insèrent. Lanalyse met en évidence trois étapes qui permettent de rendre compte des particularités de la dynamique dévolutive dans les APSA non scolaires. Lintérêt que nous portons aux formes d'intervention construites en dehors des traditions scolaires nous amène à prendre une distance qui en retour permet de mieux apprécier les pratiques scolaires plus familières.
\end{abstract}

Mots-clés : Dévolution, milieu, institution non scolaire, activité physique sportive et artistique, savoirs du corps.

\section{Introduction}

\section{La dévolution, élément de description de l'action du professeur}

Le concept de dévolution a été initialement proposé par Brousseau (1982) en didactique des mathématiques pour caractériser la manière dont l'enseignant collaborateur d'une ingénierie didactique, «fait en sorte que les élèves interagissent avec le milieu de la situation adidactique choisie » (PerrinGlorian et Hersant, 2003). Ce concept a par la suite, été travaillé pour modéliser l'action du professeur en situation de classe ordinaire. Lidée de dévolution a alors pris place dans un système de catégories qui permettent de décrire les modalités courantes de construction du savoir dans la classe (Sensevy, 2002) : définir, dévoluer, réguler, institutionnaliser.

À la suite de Sensevy (2007) nous entendons : par "définir » une situation, le fait de transmettre des règles que les élèves doivent comprendre nécessairement, au moins à un premier niveau, pour s'engager dans la situation; par « dévoluer », celui de faire en sorte que les élèves assument, dans la situation, la responsabilité d'affronter un problème; par « régulation», celui d'influer sur la production de stratégies gagnantes par les élèves; par « institutionnaliser », celui de reconnaître et fixer dans leur activité les comportements légitimes, adéquats à la situation. Ainsi l'idée de dévolution, inscrite dans ce système de notions, participe à l'élaboration d'une «théorie » de l'action du professeur. Notre propos loin d'être prescriptif (l'action du professeur « doit » contenir de la dévolution) se veut descriptif (« il peut être utile » de considérer l'action du professeur comme de la « dévolution »), car certains événements ou comportements en classe, sans cette description, resteraient non vus.

\section{Situations à la limite du didactique et portée du concept de "dévolution "}

La théorisation didactique a été élaborée en grande partie à propos de situations mathématiques scolaires. Dans ce contexte, il est possible d'appréhender les opérations sur et à partir d'objets mathématiques lors d'analyses a priori, sans prendre en compte les processus qui génèrent ces opérations. Or, d'autres situations scolaires prennent pour référence des pratiques sociales où circulent des savoirs 
de nature et d'usage nettement distincts de ceux produits en mathématiques. La «théorie» s'est efforcée d'intégrer cette spécificité des situations didactiques suivant les disciplines scolaires enseignées. Toutefois certaines situations de transmission de savoirs sont à la limite du didactique. Elles ne sont pas explicitement porteuses d'une intention d'enseigner. Les interactions professeur/élèves y sont en grande partie contingentes. Ces situations existent dans des institutions où l'apprentissage de savoirs n'est pas la finalité officielle et plus encore, dans des institutions non scolaires de transmission. La spécificité de ces situations-limites rapportées aux savoirs qu'elles mettent néanmoins en œuvre et aux formes de leur transmission, nous amènent à réfléchir sur la généricité conceptuelle de la catégorie « dévolution ». Nous l'utilisons ici pour aborder un corpus où l'enseignement-apprentissage concerne des activités physiques, sportives et artistiques (APSA) non scolaires.

Le concept de dévolution, comme les autres concepts issus des recherches centrées sur l'enseignement des mathématiques, a confirmé son intérêt décisif pour traiter la complexité de situations d'enseignement scolaire différenciées. Nous souhaitons opérer une avancée dans l'étude des situations de transmission-acquisition de savoirs, dans des pratiques sociales non scolaires, où interagissent deux ou plusieurs individus (sans être un collectif classe). L'action didactique décrite par le modèle conceptuel au degré le plus spécifique (circonscrit à un contexte précis) doit pouvoir l'être aussi à un degré plus élevé de généricité, permettant la comparaison des phénomènes didactiques issus de pratiques sociales différentes. Une ouverture et une vigilance anthropologique et épistémologique (au sens de Bachelard, 1938, 1977) nous apparaissent nécessaires à la constitution d'une part, des didactiques disciplinaires et d'autre part, de la didactique comme champ scientifique autonome.

Comment se manifeste la dévolution dans des situations non scolaires d'activités physiques : une double spécificité?

Notre étude s'attache à relever différentes manifestations de la dévolution dans trois situations d'APSA non scolaires, dissemblables à la fois par leur nature et par le mode d'intervention pratiqué. Il s'agit de: (i) l'entraînement athlétique dans le sport de haute performance; (ii) la pratique de la danse en centre spécialisé pour adolescents autistes; (iii) l'activité aquatique d'éveil dans une association pour « bébés nageurs». Ce choix de situations non scolaires d'APSA, organisé en vue d'une étude contrastée, renvoie aux questions de généricité et de spécificité qui nous animent à propos de la dévolution. Nous donnons ci-dessous une brève description des spécificités institutionnelles, finalités et objectifs des différentes situations, avant de cerner un moment significatif de leur fonctionnement au quotidien qui a retenu notre attention.

\section{Séances d'entraînement avec un athlète de haut} niveau

Les séances d'entraînement athlétique se déroulent en début de saison compétitive et concernent un entraîneur et son athlète lanceur de disque, de niveau national. L'intervention de l'entraîneur, habituellement réalisé sur un mode magistral et ostentatoire, peut être globalement qualifiée de "prescriptive ». Ces propos, le plus souvent succincts et allusifs, consistent en des remarques ponctuelles sur des points techniques précis. Ils sont parfois assortis de mimes illustratifs qui viennent combler les « creux » d'un discours (inévitablement) parcellaire sur la mécanique gestuelle (Vivès et Vigarello, 1983, 1986, 1989). Létude porte sur une séance où ses interventions contrastent en partie avec ce mode usuel. Lathlète ayant participé aux championnats de France hivernaux, effectue sa reprise d'entraînement de mi-saison, après une période de repos d'environ trois semaines. Le thème de travail fait partie des standards de l'entraînement du lancer de disque. Il s'agit pour l'athlète d'amener son pied gauche le plus vite possible au sol à la fin du geste de lancer, en empêchant une rotation " trop » importante du corps autour du pied droit. L'athlète rencontre des difficultés sur lesquelles notre attention s'est focalisée.

Séances de danse avec trois adolescents autistes

Les séances de danse se déroulent dans un centre d'éducation spécialisé pour adolescents atteints 
d'autisme. Le professeur d'EPS en charge de trois adolescents, sans être spécialiste de l'activité danse, collabore à un travail d'ingénierie didactique où il a été décidé de faire pratiquer à ces adolescents la danse contemporaine. Le choix de ce type de danse est fonction du handicap autistique dont les deux principales manifestations sont les suivantes : troubles du développement qui touchent les interactions sociales et verbales, perturbées en quantité et en qualité; abandon de toute activité du monde social dès que s'interrompt la stimulation venue de l'extérieur. L'objectif du professeur, accordé à ceux de l'ingénierie, est d'amener les adolescents à développer une motricité de communication pour leur permettre de « se faire comprendre » d'autrui et de " comprendre » autrui sur un plan affectif. Le thème de travail (en lien avec la pratique de la danse contemporaine) concerne les contacts corporels entre les danseurs en mouvement. Ces contacts sont structurés suivant les différentes «énergies » de temps, flux, poids et espace (définies par Laban, 2003). Leur utilisation prend pour fil directeur l'expression des émotions et sentiments : les adolescents et l'enseignant « jouent » sur ces contacts. Lors d'une séance du début de cycle, nous sommes attentifs à la manière dont ensemble ils attribuent une signification émotionnelle et sentimentale aux gestes de contact émis.

\section{Séances d'éveil aquatique avec une fillette de trois ans}

Nos observations se centrent sur un club de « bébés nageurs ». La finalité principale de cette association est de créer une activité familiale où le jeune enfant développe aisance et autonomie dans l'eau. L'organisation des séances d'activité aquatique d'éveil est prise en charge par une équipe d'animateurs constituée de bénévoles formés ou non par la Fédération des Activité d'Éveil et de Loisir (FAAEL) et de professionnels titulaires du Brevet d'État de Secours et Sauvetage des Activités Nautiques (BESSAN). Le matériel est agencé de manière évolutive en fonction des tranches d'âge (deux premiers groupes : 6-18 mois; deux groupes intermédiaires: 18-36 mois; deux groupes : 3-6 ans). Une fois le matériel en place, les animateurs se mettent à disposition des parents et interviennent auprès d'eux de manière ponctuelle et individuelle en fonction de leur demande. Les parents accompagnent les évolu- tions de leur enfant dans l'eau, en fonction de leur propre vécu passé ou actuel de «nageur» et selon un fonctionnement didactique qui leur est propre. Lapprentissage du plongeon canard est présent chez les trois familles que nous observons. Dans le cadre de cet article nous choisissons une séance mettant en scène une petite fille $(\mathrm{Az})$ alors âgée de trois ans, accompagnée par ses deux parents. À ce stade de son adaptation au milieu aquatique Az explore la petite profondeur en descendant dans le bassin «par les pieds » et non par la tête. Elle demande régulièrement à son père ou à sa mère de l'aider pour aller chercher de petits objets immergés dans de plus grandes profondeurs.

Étudier des situations aussi variées, dans des institutions non scolaires différentes où elles se développent, permet une vision élargie des pratiques sociales d'APSA comme lieux de transmission et d'acquisition de savoirs. Nous examinons dans quelle mesure et sous quelle(s) forme(s) l'acte dévolutif se décrit, au sein de ces pratiques sociales. Nous prendrons en compte deux niveaux de généricité : le premier permettant de comparer toute pratique d'intervention, scolaire et non scolaire; le second permettant d'ébaucher un modèle commun aux interventions non scolaires. Il s'agit également d'envisager une spécification de ce concept selon les différentes institutions observées.

\section{Cadre théorique}

\section{Dévolution et milieu}

L'objet de notre étude, les transformations motrices dans l'activité corporelle, en contexte non scolaire, constitue la première particularité de nos investigations. La seconde réside dans l'emprunt du concept de dévolution jusque-là utilisé pour décrire l'action du professeur à l'école, et son importation dans un cadre institutionnel autre, celui des APSA non scolaires. Éprouver le concept de dévolution dans un contexte non scolaire est particulièrement intéressant car nous pourrions penser spontanément qu'un tel contexte suscite un investissement « libre » et volontaire de l'apprenant dans des situations ouvertes et hors contrainte. S'il peut sembler évident que la nécessité de dévolution est plus impérieuse 
dans des contextes non scolaires, il nous faut en identifier les formes. Par ailleurs importer le concept de dévolution nous conduit à associer la notion de dévolution à celle de milieu, qui actualise nécessairement les pratiques corporelles. Nous considérons donc de manière très précise la nature dévolutive des relations didactiques à travers les caractéristiques du milieu dans lequel elles s'insèrent.

La mise au point du concept de milieu, en déplaçant le regard porté sur les actes d'enseignementapprentissage appréhendés essentiellement selon une approche psychologique, a " dé psychologisé » les comportements des acteurs en situation. Les études sur le milieu entre (medius) le professeur et l'élève, se décentrent des sujets eux-mêmes pour étudier les liens établis par ces sujets avec les savoirs en situation. Par ailleurs elles donnent le primat à une épistémologie externaliste qui tient compte des influences $\mathrm{du}$ milieu sur la constitution des savoirs. Lidée de milieu s'est donc développée comme un élément décisif des productions des élèves, de leur réussite ou de leur échec au sein de l'institution scolaire. Elle est envisagée, d'une manière large, comme une réalité à partir de et sur laquelle agit l'élève. Le milieu contraint l'action en même temps qu'il constitue une ressource pour elle (Chevallard, 1992). Il en est le substrat puis le produit, à la fois objet des transactions didactiques ${ }^{1}$ et condition de possibilité de ces transactions.

\section{Les milieux}

Les travaux de Perrin-Glorian (1999) établissent certaines distinctions. Le milieu est caractérisé vis-à-vis des actions présentes de l'apprenant, selon son rôle qui peut être " antagoniste » de ses actions (dans la théorie des situations de Brousseau, 1986) et/ou "allié » (dans la théorie anthropologique du didactique chez Chevallard, 1992). Il arrive ainsi que l'action des trois intervenants empêche l'expression de certains comportements usuels des apprenants de manière à permettre, à terme, l'expression d'une motricité efficace. Une autre distinction (PerrinGlorian, ibid.) cerne le statut ontologique du milieu : « matériel » ou « objectif» (c'est-à-dire indépendant du face à face enseignant-élèves), ou « symbolique » (accédant au statut de milieu à travers une activité de symbolisation). Cela nous porte à prendre en compte des éléments de consignes comme instigateurs d'un milieu pour l'action de l'apprenant. Ces distinctions autorisent à parler de « milieux » au pluriel.

\section{Milieu rétroactif pour une action motrice "authentique"}

Se pose alors un problème fondamental en matière d'éducation motrice : comment fabriquer des milieux « adéquats » à la transformation par les élèves de leur motricité habituelle. Le travail de l'enseignant (impliqué dans une ingénierie didactique) consisterait à créer un milieu pour l'action motrice de l'élève, milieu qui serait également fortement rétroactif. Les rétroactions du milieu déclencheraient la mise en jeu des processus moteurs adaptatifs et en conséquence l'émergence des réponses attendues par le maître. Les situations dites adidactiques (c'est-à-dire où disparaît l'intention manifeste d'enseigner, dans le vocabulaire de Brousseau, 1998) sont considérées comme les plus aptes à favoriser les acquisitions visées, même si elles ne sont pas suffisantes en elles-mêmes. Elles donnent l'illusion à l'apprenant de " jouer contre la nature ", d'être enseigné "par les choses », et d'échapper ainsi à la « figure du professeur » (Chevallard, 1989) et à son bon vouloir. Il s'agirait alors pour lui de créer un milieu « a-institutionnel » (Chevallard, 1989, cité par Perrin-Glorian, 1999). Dans ce type de situation, l'élève serait tenu d'agir « de son propre mouvement, proprio motu » et de « se prendre au jeu », c'est-à-dire « d'accepter de jouer en première personne le jeu de savoir $»$ (Sensevy, 2007).

Les objets matériels du milieu en APSA

Dans le cadre d'une ingénierie, l'élève est placé face à un problème (au sens général de ce terme, dont la solution échappe aux élèves) par sa confrontation à un milieu. Celui-ci est d'une part, suffisamment ouvert pour permettre l'expression chez l'élève d'une exploration des possibles (arrière-fond de savoirs anciens) et d'autre part, suffisamment fermé (les « choses» résistent) et rétroactif (il faut «se rendre à l'évidence : la façon de s'y prendre pour régler le problème ne convient pas »). Ce sont les rétroactions du milieu qui, avec l'aide de l'action de l'enseignant, forceront l'élève à des régulations en retour, qui l'amèneront à réfuter les stratégies inopérantes et l'orienteront vers la production de la solu- 
tion attendue. Celle-ci sera alors institutionnalisée. Le milieu des APSA comprend, en grande part, des objets matériels utilisables par l'élève et sur lesquels il exerce ses actions. Les modalités de leur utilisation apparaissent au gré des rétroactions ou sont induites par un rapport à ces objets, constitué au fil de la relation didactique (contrat et mémoire didactiques, dans le vocabulaire de Brousseau et Centeno, 1991). Modéliser l'apprentissage en APSA sous la forme d'une adaptation pour laquelle les rétroactions du milieu jouent un rôle décisif amène donc à penser les objets matériels dans un milieu qui fait sens aux yeux des élèves, d'où leur nécessaire acculturation préalable à ces objets.

Toute l'économie de la dévolution, en tant que produit d'ingénierie didactique, repose sur une grande complexité préparée en amont de l'action de l'élève en situation effective. Il pourrait alors être tentant d'envisager la dévolution uniquement au niveau de la conception de l'ingénierie, ce qui constituerait une vision réductrice de la dévolution. Nous souhaitons faire la lumière sur la dévolution en montrant qu'elle est une nécessité constitutive des relations didactiques in situ. Nous l'envisagerons donc de manière diachronique à travers ses effets sur les apprentissages et, plus largement, sur les actions que les apprenants accomplissent en retour sur le milieu.

\section{Milieu potentiel, milieu activé}

S'engage alors une réflexion sur une acception plus large de la notion de milieu, incluant les influences du contrat didactique et du vécu didactique de l'apprenant. Les travaux récents en didactique se sont engagés dans cette voie pour expliquer les « bifurcations » des élèves. Ils distinguent alors différents milieux « emboîtés » (Comiti, Grenier et Margolinas, 1995). Sans nier l'utilité d'externaliser l'étude $\mathrm{du}$ milieu, il nous semble heuristique d'y inclure plusieurs dimensions liées au passé didactique de l'apprenant et, plus largement, à son vécu personnel actuel. Il s'agirait ainsi pour nous de dépasser la vision selon laquelle « certains savoirs présents dans le milieu peuvent ne pas être reconnus par les élèves parce que leurs connaissances ne le leur permettent pas» (Perrin-Glorian et Hersant, op. cit.). Ne pourrait-on pas tout autant affirmer que l'échec des élèves dans la situation proviendrait d'un déficit au niveau de l'épistémologie du professeur? Il est possible que le travail professoral ne maîtrise pas l'ensemble des conditions d'acquisition de tel ou tel savoir au regard des acquis des élèves. Nous admettons l'utilité de parler d'une (in)adéquation de la situation (adidactique par exemple) et d'opter pour une définition extensive de la notion de milieu en acceptant l'idée que le milieu pourrait inlure des dimensions composites comme le vécu didactique de l'élève, ses désirs et motifs d'agir, etc. Le milieu comprendrait plus classiquement tout ce que l'on pourrait objectiver en termes de ressources et contraintes, «données » par l'action didactique de l'enseignant (Perrin-Glorian parle alors de milieu « objectif »). Une étude ascendante et « rétrodictive » (au sens de Veyne, 1978) des relations didactiques nous semble indispensable pour mettre à jour, empiriquement, l'évolution in situ de ces différentes dimensions du milieu dans lesquelles s'insère le travail dévolutif.

\section{Milieu pourvoyeur d'actions conjointes enseignant-élèves}

En outre, nous prenons en compte les progrès de la modélisation du milieu, accomplis grâce à l'expansion des didactiques disciplinaires et au développement de l'approche comparatiste en didactique. Comme nous l'avons déjà évoqué, les analyses a priori, en didactique des mathématiques, ont permis de rendre compte des différentes opérations possibles sur les objets du milieu objectif. Aujourd'hui, les travaux sur "l'action didactique conjointe " (Sensevy, 2007) explicitent la dimension fortement réciproque des interactions enseignant-élèves, ce qui oblige à penser les savoirs non pas seulement en vision externe à l'action mais également tels qu'ils sont actualisés au cœur des échanges et déployés dans la durée. Dans le prolongement de la théorie de l'action didactique conjointe, envisager les coactions enseignant-élèves comme fondements de la création et du remaniement du milieu semble heuristique. En outre, l'intégration du vécu, des actions d'autrui, des bifurcations engage les recherches didactiques dans une perspective ouverte aux données temporelles, situées et émergentes du milieu. Il nous semble donc utile de porter un regard anthropologique fin sur la détermination réciproque des co-actions enseignant-élève(s) et les transformations du milieu. 


\section{Les propriétés corporelles : un élément-clef dans la didactique des APSA}

Par ailleurs, notre étude porte sur les transformations des pratiques corporelles. Or l'activité motrice du corps est à la fois créatrice d'effets et utilisatrice de ces effets. Dans le cadre des techniques corporelles, il est tout autant «agi » qu'il agit. Il nous semble alors important d'inclure la spécificité de l'activité motrice dans la définition du milieu : le pratiquant, en agissant par son corps et sur son corps, redéfinit à chaque instant du déroulement gestuel, un éventail de possibles pour «l'aval moteur ». Les techniques corporelles peuvent ainsi être caractérisées par leur pouvoir catalytique de réactions en chaîne entre le corps actant et le corps agi. Les « savoirs du corps » sont des objets de sensation et de connaissance parfois difficilement formalisables, inscrits dans la temporalité du geste. Les savoir-faire comme produits sont alors difficilement séparables des mécanismes en jeu dans leur production. Ils renvoient à des processus moteurs souvent incommensurables, pour lesquels la complexité de la régulation de l'acte moteur est difficile à saisir. Nous remarquons, par ailleurs, qu'il n'y a pas une exclusivité de la transmission des savoirs corporels, qui serait réservée à l'EPS. Au contraire, la diffusion des techniques corporelles s'effectue au cœur de diverses institutions où les techniques sont inventées, mises au point ou utilisées, de manière plus ou moins formalisée et standardisée.

Cet ensemble de questions nous amène à poser le problème de la constitution d'une didactique des activités physiques, sportives et artistiques (APSA), en tant que pratiques d'intervention et discipline scientifique se préoccupant de ces pratiques. Du point de vue des pratiques didactiques, l'intervenant ne peut pas être au clair sur la totalité des déterminants de l'acte moteur. Tout au plus possède-t-il des "modèles de la pratique " (Marsenach, 1991) qui orientent peu ou prou son intervention auprès des élèves de manière à favoriser les acquisitions attendues. Ces attentes ne sont d'ailleurs pas toujours facilement formalisables, en témoignent notamment les difficultés rencontrées par les professeurs d'éducation physique et sportive (EPS) pour trouver un consensus relatif à la définition des "contenus à enseigner » dans telle ou telle APSA. Si les contenus en EPS sont « les conditions que l'élève doit intégrer pour transformer ses actions », ces conditions sont « organisées pour que progressivement l'élève connaisse mieux les propriétés du milieu dans lequel il interagit, les propriétés de ses actions et les propriétés de l'outil qui lui permet d'agir, c'est-à-dire son corps » (Marsenach, 1991, p. 44). Elles renvoient à des registres d'activité très différents (conçus de manière traditionnelle en pôles cognitifs, moteur, affectif, informationnel...) pour des transformations elles-mêmes identifiables de différentes manières. Quoi qu'il en soit, l'enseignant ne peut faire l'impasse d'élucider ces conditions, s'il tient à réaliser une dévolution efficace.

Si aucune analyse ne permet de rendre totalement compte d'un savoir-faire (Guillerme, 1985), l'épistémologie de l'intervenant est inévitablement lacunaire et parcellaire. Elle l'est d'autant plus dans les pratiques non scolaires où les intervenants, peu formés aux questions didactiques, recourent à des savoirs d'intervention majoritairement basés sur l'empirie. Il semble alors incongru, tout particulièrement dans ces pratiques corporelles non scolaires, de penser les situations où la dévolution intervient comme le produit d'une ingénierie didactique. Lintervenant, quel que soit son rapport à la pratique, agit (intuitivement) sur le milieu de manière à créer des situations adidactiques propices à l'acquisition des savoirs visés. Lidée d'une dialectique média/milieu, proposée par Chevallard (2002), est ici particulièrement éclairante. Elle questionne la manière dont l'enseignant met en rapport ce qu'il propose à l'apprenant et ce qu'il veut lui faire acquérir. Ainsi, lorsque l'enseignant ne parvient pas à construire un milieu avec l'élève, il propose une médiation avec le savoir que l'élève va devoir étudier de sa propre initiative (on dit qu'il y a dévolution de l'objet à étudier). L'enseignant dirige alors cette étude, en proposant parfois des conditions qui font milieu. Les catégories du modèle de « l'action du professeur » (définir, dévoluer, réguler, instituer, Sensevy, 2002, 2007) sont-elles productives hors des corpus scolaires qui ont donné naissance à la théorisation? Le statut non scolaire de pratiques corporelles conférerait-il une spécificité à l'action dévolutive de l'intervenant? Pourrait-on y repérer des régularités inhérentes à ce type de pratique, des formes typiques de structuration de la dévolution, aussi incidentes et non voulues qu'elles puissent être?

Ainsi, l'étude des techniques corporelles au sein de trois APSA différentes nous place face à des corpus pour lesquels la complexité des «savoirs du corps » 
et le caractère fondamentalement communicationnel et donc contingent de leur transmission exigent un traitement original. Ce prolongement empirique aux études sur la dévolution, dans le cadre d'APSA non scolaires, oblige à imaginer des outils et méthodes, ayant fait leur preuve dans les corpus scolaires mais tenant compte spécifiquement des pratiques d'APSA et des institutions non scolaires dont elles dépendent.

\section{Méthodologie}

Nous avons abordé les différentes pratiques selon une même méthodologie, élaborée afin de mettre à jour le caractère dévolutif des APSA non scolaires. Notre étude postule qu'il existe une forme spécifique de dévolution dans ces pratiques par rapport aux pratiques scolaires, du fait de la nature des savoirs en jeu et des particularités de leurs lieux de transmission. Nous présenterons les aspects communs et les spécificités de la dévolution à travers trois études de cas, chacun dans une pratique différente. Les données sont issues de travaux initialement indépendants.

Les hypothèses émises conduisent à choisir des outils originaux conçus pour l'étude de la motricité humaine. Les critères d'observation retenus visent à reconstituer le fil d'une « intrigue » (Veyne, 1978) didactique. Lintrigue didactique en tant que forme descriptive a été initiée par Sensevy (2007) et travaillée dans le domaine des APSA par Loquet et al. (2007). Elle permet de réduire et condenser la pratique en un produit comportant, pour ce qui nous concerne, des moments d'adidacticité. Une démarche ascendante basée sur les trois études de cas en situation ordinaire s'impose alors et ceci en utilisant des outils méthodologiques communs aux trois études. Les trois intervenants, reconnus comme expérimentés, ont été "sélectionnés » pour l'efficacité de leurs interventions, attestée auprès des apprenants (apparition de stratégies prometteuses ou gagnantes, suivant les cas). Dans la mesure où les modalités des transactions didactiques sont en partie conditionnées par la nature des savoirs à acquérir (« savoirs du corps » en partie indicibles) mais aussi par les particularités du « collectif » d'apprentissage (communications intersubjectives quasi-duelles par nécessité), nous organiserons notre recherche autour de trois types d'analyse, didactique épistémologique et anthropologique.
Nous avons cherché à identifier tout d'abord les savoirs choisis a priori comme enjeux d'acquisition par l'intervenant (entretiens semi-directifs) avant de nous intéresser au « déjà-là » des apprenants : ce qu'ils savent faire relativement au thème choisi par l'intervenant. Notre attention s'est ensuite portée sur les moyens mis en œuvre par l'intervenant pour faciliter les acquisitions par l'apprenant et enfin les effets de cette mise en œuvre sur sa motricité et ses savoirfaire (observations in situ). Un triplet de catégories mis au point par les recherches didactiques permet de rendre visibles de manière générique les techniques d'intervention et la manière dont les apprenants agissent conjointement avec l'intervenant. Peuvent ainsi être distinguées (Sensevy, 2002) les techniques mésogénétiques (relatives à la genèse des savoirs dans le milieu), topogénétiques (relatives au partage entre le professeur et l'élève, des responsabilités vis-à-vis des savoirs) et chronogénétiques (relatives à l'avancée des savoirs dans la classe).

Une description mésogénétique pourra être attentive à la manière dont l'intervenant introduit dans le milieu une « signification » d'un certain ordre grâce à un mouvement, un geste, un énoncé ou une manipulation d'objets, ou encore en attirant l'attention sur un aspect de la production de l'élève. Utiliser la catégorie " technique mésogénétique » pour décrire une séquence didactique précise, consiste donc à étudier le contenu des actions des uns et des autres et voir ce qui, dans les interactions, peut agir sur la configuration des possibles et des nécessaires pour l'action de l'apprenant. Une description topogénétique, quant à elle, mentionne les acteurs de cette génèse. On se demande quelle est la part d'action de l'intervenant et de l'apprenant, à leur place respective dans le milieu : par exemple qui a introduit tel objet ou telle manière de faire, qui a validé tel ou tel comportement. Enfin, une description chronogénétique se concentre sur l'introduction d'éléments nouveaux dans le défilement du temps (Chevallard, 1992) et sur l'effet de cette nouveauté dans les transformations motrices de l'élève. Considérer ensemble ces trois catégories permet d'éclairer avec plus de finesse le phénomène dévolutif qui nous interroge. La dévolution sera mise en lumière à l'aune des responsabilités (topogénèse) prises à tour de rôle par les acteurs; sa perception sera améliorée par l'analyse du passage, à certains moments, de l'ancien au nouveau, d'un contenu de motricité à un autre (chronogénèse); et sera nourrie 
enfin de façon privilégiée par l'analyse des objets qui trouvent sens dans l'environnement commun intervenant-apprenant (mesogénèse).

Notre recherche assure l'articulation des données issues des entretiens (traduites en termes d'intentions et d'attentes de l'intervenant) avec celles obtenues par l'observation de terrain (en termes de co-actions). Nous avons donc opté pour un recueil de données empiriques basé sur le film de l'ensemble des séances d'un cycle d'apprentissage (marquant une centration sur un enjeu d'acquisition particulier ou une période-clé de l'année). Sont pris en compte l'ensemble des interactions entre le (ou les) intervenant(s) et le (ou les) apprenant(s), et l'ensemble des décisions prises par le (ou les) intervenant(s). Suite à ces recueils in situ, les données sont transcrites, en restituant la chronologie du déroulement, sous deux formes associées : les paroles et les actions. Un événement remarquable a été sélectionné dans chacun des corpus et isolé parmi d'autres événements possibles. Un événement est dit remarquable s'il cristallise l'interaction didactique, c'est-à-dire s'il comporte une multitude d'échanges de natures diverses autour des acquisitions visées par l'intervenant. Le traitement de ces données, après description minutieuse, a tout d'abord été centré sur les rapports d'occurrence entre les actions de l'intervenant et celles de l'apprenant. Nous avons repéré les transformations motrices relatives au thème travaillé (identifié à partir des consignes et intentions didactiques annoncées en entretien). Ainsi, le thème du « retour de la jambe gauche » a centré l'attention du chercheur sur l'action du membre inférieur opposé au bras lanceur, dans la mécanique du geste athlétique; celui de "l'expression des émotions », sur les gestes de consolation et les mimiques du visage chez les autistes; celui du « déplacement aquatique », sur le critère de l'horizontalité du corps comme condition de transformation de la motricité.

Nous avons ensuite distingué dans l'ensemble des gestes et attitudes de l'apprenant, ceux qui sont efficaces (ou prometteurs d'efficacité) de ceux qui échouent : au plan des rapports sociaux pour les autistes ou au plan biomécanique pour l'apprenti nageur ou l'athlète. Ces gestes et attitudes sont ensuite rapportés aux interventions du professeur, parent ou entraîneur.
Nous avons alors construit le « récit » des interactions d'un point de vue didactique, et dégagé des traits pertinents (Schubauer-Leoni et al., 2007) établissant des liens entre les interventions et les actions afin de voir en quoi, à certains moments, des interventions sont plus ou moins efficaces. La catégorie « milieu » dont nous postulons la nécessité pour décrire la valence dévolutive de l'intervention, s'est avérée indispensable; elle s'est affinée et spécifiée au regard de notre objet d'étude en situation non scolaire. Nous avons été amenés à en distinguer différents, les uns induits par l'action didactique de l'intervenant, les autres activés par l'usage moteur que l'apprenant fait de son corps. Cette analyse nous conduit à étudier les composantes a-didactiques des situations en fonction de l'évolution topogénétique de ces situations. Nous avons donc constitué des trames décrivant les relations successives entre les "états » des situations spécifiques (fonction de «l'état» des transformations motrices) et les techniques sur lesquelles repose le travail de l'intervenant, en particulier celles qui portent sur les milieux. Des aspects génériques ont pu être identifiés dans l'évolution des rapports entre les techniques de l'intervenant et ses effets sur les objets qui prennent sens dans les milieux. Nous avons alors isolé, toujours de manière générique, les moments a-didactiques tout en recherchant les liens avec leurs conditions d'apparition, de manifestation, de stabilisation pour comparer ces aspects de généricité avec les moments a-didactiques rencontrés dans l'institution scolaire ordinaire.

\section{Résultats}

Létude de chaque cas caractérisé par un mode dévolutif non scolaire, nous a permis de dégager des aspects génériques de dévolution communs aux APSA non scolaires. Un de ces aspects concerne la temporalité : la dévolution se réalise en trois grandes étapes successives dont on peut extraire certains traits communs. La présentation des résultats est organisée autour de la description de ces étapes.

\section{Première étape : création d'un milieu pour l'action, l'adidacticité}

Nous dégageons une première caractéristique commune aux trois corpus. Elle concerne les conditions de création d'un milieu propice à l'activité 
motrice exploratoire des apprenants et favorable à la « mise en dévolution ». Un tel milieu est observé alors qu'au préalable, le milieu initial (et habituel) se trouve soit saturé en consignes, soit au contraire extrêmement lâche et pauvre en consignes. Ainsi dans le cas de la pratique sportive de haut niveau, la part d'action de chacun, dans le couple formé par l'entraîneur et l'athlète, correspond au fonctionnement suivant : pendant une durée importante, l'entraîneur en "position de surplomb » (SchubauerLeoni et al., 2007) charge l'athlète de suivre des consignes nombreuses et répétées sur des points techniques précis. A contrario les séances de danse avec les adolescents autistes et l'activité aquatique d'éveil avec les tout jeunes enfants font apparaître un partage d'action où l'intervenant suspend les consignes, le milieu initial apparaissant peu contraignant. Nous présentons la manière dont ces phénomènes s'actualisent dans les trois cas, à travers le récit de chaque pratique, complété par des éléments d'analyse a priori permettant d'informer les descriptions produites.

\section{L'entraînement athlétique du lancer de disque}

Cette activité consiste à lancer un disque métallique de deux kilos à partir d'un cercle cimenté d'un diamètre de $2,50 \mathrm{~m}$. L'athlète est libre d'évoluer comme il l'entend à l'intérieur de ce cercle, à condition d'en ressortir par l'arrière une fois le disque entré en contact avec le sol. La technique considérée comme étant la plus efficace consiste à : partir de dos, $\mathrm{au}$ « fond » du cercle de lancer, puis effectuer une volte, c'est-à-dire un tour et demi sur soi-même en avançant dans le cercle. En fin de geste, est réalisée ce que les athlètes et les entraîneurs nomment la phase de double appui : le pied gauche reste pratiquement immobile au sol (point d'appui fixe) et, grâce à l'action du pied et du membre inférieur droits, (et à la vitesse de déplacement accumulée), « reçoit» de plus en plus de pression. Lathlète, grâce à cette action dissociée des appuis, met sous tension (en les allongeant) les muscles et chaînes musculaires dont la contraction permet ensuite d'accélérer spécifiquement l'engin. Lathlète doit donc coordonner, des membres inférieurs « vers» les membres supérieurs, une succession de contractions et d'étirements musculaires (en sachant par exemple que la contraction des muscles extenseurs des membres inférieurs permet, du fait du pivot et de l'avancée du bassin, d'étirer les muscles rotateurs du tronc et qu'ensuite, la contraction de ceux-ci permettra l'étirement du grand pectoral). À un fonctionnement neuromusculaire optimal, l'athlète doit faire correspondre des principes mécaniques : ainsi, par exemple, il doit chercher à obtenir un chemin d'accélération (distance parcourue par le disque au cours de laquelle il est accéléré) le plus long possible et, durant ce « temps » de parcours, à appliquer une accélération constante (les variations d'accélération étant mécaniquement inefficaces et très coûteuses sur le plan énergétique). Lorsque l'athlète est sur un appui (droit ou gauche), il peut accélérer son corps dans son ensemble. Les transferts dits «étagés » de forces (d'accélérations, de vitesses...) des membres inférieurs vers les membres supérieurs ne sont possibles qu'à condition d'avoir deux appuis au sol. C'est pourquoi les entraîneurs et leurs athlètes essaient souvent de créer les conditions d'une pose « terminale » de l'appui gauche la plus rapide possible. Cela permet de débuter le double appui et d'enclencher les actions entraînant l'accélération spécifique du disque au plus tôt. Une avance optimale du bas du corps est ainsi possible, générant les étirements et trajets d'accélération les plus amples (selon les possibilités de l'athlète, liées à son gabarit et à ses qualités musculaires). Le "retour » du membre inférieur gauche en vue de poser le pied gauche au sol constitue donc un des standards de l'entraînement technique au lancer du disque.

Létude porte sur une suite de sept séances d'entraînement, en période de reprise technique, l'athlète - de niveau national - n'ayant pas lancé le disque depuis un mois environ. 338 lancers ont été dénombrés pour un total de 451 consignes abordant des points techniques. On compte une "saturation didactique " moyenne de 1,33 consigne technique par lancer. Cette valeur est nettement supérieure à la saturation moyenne sur l'ensemble de l'année. Durant la séance observée (deuxième séance), l'entraîneur cible ses interventions sur la dissociation des trains inférieur et supérieur à partir du travail des appuis au sol servant à accélérer le disque en phase finale. Les situations étudiées (comprises entre les minutes 9'35 et 14'17) s'organisent autour du thème de l'action du membre inférieur gauche suite à la reprise de contact du pied droit au centre du plateau de lancer, préparatoire au double appui final. 
Les mouvements de flexion/extension, adduction/abduction et supination du membre inférieur gauche visent à amener le pied gauche au sol le plus vite possible tout en conservant un retard du haut du corps avant le début de l'action du membre inférieur droit. En empêchant le corps de tourner autour du pied droit suite à sa reprise de contact, ce mouvement permet de créer les conditions mécaniques (éloignement du disque) et musculaires (étirements) d'une accélération efficace du disque en fin de geste.

Lathlète tend, à la reprise de contact de l'appui droit au sol, à réaliser une rotation globale de l'ensemble du corps autour de cet appui : il continue à tourner autour de son appui droit, de manière «monolithique » (presque pas de dissociation des ceintures pelvienne et scapulaire). Le contact de l'appui gauche au sol se réalise lorsqu'il se trouve de face par rapport à la surface de réception du disque, d'où une mise sous tension musculaire nettement inférieure à celle des standards de réalisation technique, caractérisés par une pose du pied gauche alors que l'athlète se trouve encore orienté de dos par rapport à la direction du lancer (cf. ci-dessus).

Les conditions biomécaniques d'un travail dissocié des appuis n'étant pas idéales, l'athlète réagit de manière syncrétique (globale, non dissociée) et explosive sur ses membres inférieurs en procédant à leur extension violente et simultanée dès la pose du pied gauche.

Lentraîneur met en place une situation en début de deuxième séance afin de rapprocher la motricité de l'athlète des standards de réalisation définis à haut niveau concernant l'action du membre inférieur gauche ("pour l'empêcher d'être haut et de partir tout de suite sur son côté gauche "; "C'est un peu la moto que tu veux démarrer, le kick, et hop tout de suite tu tapes [...] C'est pour éviter en plus...c'est pour éviter, si tu mets tes deux genoux au même niveau, de monter sur tes appuis aussi. » - entretien post séance avec l' entraîneur). Il s'agit pour l'athlète de partir sans élan, de dos à la direction du futur lancer, équilibré sur le pied droit, puis d'accélérer la pose au sol du pied gauche, tout en conservant les épaules en retrait, avant d'agir avec le MID pour poursuivre le geste. L'entraîneur l'annonce comme suit :

«Ce qu'on va se contenter de faire/c'est d'accélérer la jambe gauche//peu importe comment tu l'accélères ça m'est égal tu vois?/hein?/tu accélères/tu accélères ta jambe gauche/et tu travailles sur ta jambe droite! »

Il associe un mime à cette consigne orale, deux formes d'interventions qui, l'une et l'autre, «masquent» une grande partie du mouvement escompté du membre inférieur gauche. L'entraîneur en effet ne montre que la posture de départ (en appui sur le pied droit, les deux membres inférieurs fléchis très proches l'un de l'autre) puis la fin du mouvement du membre inférieur gauche (à partir du moment où le pied gauche est à environ vingt centimètres audessus du sol, au « bout » du cercle de lancer).

De plus la situation est introduite en quelque sorte " par défaut », car elle intervient à la suite d'un ensemble de situations didactiques insuffisantes pour orienter l'athlète de manière décisive vers la réussite. Lors de ces situations, l'entraîneur abordait, de manière analytique, un par un, des points techniques précis. Lathlète réussissait à conformer son geste aux attentes techniques immédiates, annoncées par l'entraîneur dans ses consignes. Toutefois, il ne parvenait pas à intégrer, dans un geste global, l'ensemble des points visant à l'actionefficace du membre inférieur gauche, suite à la reprise de contact du pied droit au centre du plateau. En conséquence, les « entrées didactiques » de l'entraîneur se sont multipliées selon la même stratégie (points techniques abordés successivement), sans apporter de réussite. Ainsi, lors de la séance précédente, l'entraîneur, au cours d'une série de lancers « complets » de l'athlète, précise à l'athlète (temps : 15’00, 5le tour de parole), en mimant une fin de geste analogue à celle décrite précédemment : " ton genou gauche vient dépasser ton genou droit//ici/ donc là y'a vraiment une translation qui se crée/let à partir du moment où ton genou gauche a dépassé ton genou droit/tu le ramènes au sol//et/la pose ferme de ta jambe gauche entraîne la rotation de ta jambe droite/par torsion/là regarde tu//t'as pas le choix si tu veux//t'es obligé de tourner//donc c'est ça qu'il faut ressentir! "

Dans cet ensemble de situations où prédomine l'ostension par l'entraîneur, à travers mimes et consignes, la situation décrite ci-dessus (deuxième séance) prend un caractère dévolutif. En effet, l'entraîneur, suite aux échecs répétés de l'athlète, mime le geste attendu mais en en cachant volontairement la partie-clé, vue comme solution au problème de la reprise efficace de l'appui gauche. Cette volonté, 
qui semble manifeste au regard de l'association du mime et de la consigne, est confirmée par un entretien d'autoconfrontation: "Bon là je ne lui montre pas mais j'essaie de l'amener à le faire. ". Il esquisse la posture initiale du geste attendu puis l'accélération terminale du pied gauche, qui amène ce pied au sol. Il engage ainsi l'athlète à réaliser un mouvement qu'il ne présente pas en entier (c'est le premier type d'action didactique de cette sorte). Cette présentation associe une consigne : accélération du pied gauche avant l'action du membre inférieur droit, et un geste mimé : accélération du pied gauche et posture attendue à l'entame du DA. L'entraîneur laisse apparaître l'état final du geste (configuration finale comme produit des actions présidant à son obtention) suite aux actions (masquées) qui y auront été portées.

L'entraîneur adopte ainsi une autre stratégie d'ostension.

\section{L'éducation des autistes par la danse}

L'enfermement et l'absence de communication caractérisent l'autisme. L'activité danse, dont l'essence est la communication, permet de « mettre en contact » des adolescents atteints d'autisme avec une culture physique d'expression et de communication, voire de les faire « entrer dans » cette culture, en visant progressivement leur adaptation et leur socialisation. Un des objectifs de cette pratique est d'affronter l'obstacle d'expression-communication (la résistance des adolescents aux transformations est sur ce point particulièrement forte) par le biais d'un travail sur les contacts, les porters, en utilisant comme fil directeur les émotions et sentiments ressentis. Alors que certaines choses de la vie, comme ressentir les intentions et sentiments d'autrui, sont apprises précocement de façon «naturelle » chez une personne valide, chez une personne autiste les modalités d'apprentissage doivent se faire par voie cognitive explicite et « volontaire ». Parler d'apprentissage est essentiel pour ces personnes afin qu'elles aient la possibilité de s'adapter à l'environnement qui les entoure (Frith, 1992).

Lambition de la situation d'enseignement présentée ici (extraite de la troisième séance ellemême inscrite dans la réalisation d'une ingénierie didactique), est d'apporter aux adolescents autistes un vocabulaire corporel d'expression (ou un élargissement du langage corporel dont ils disposent) et une précision dans les énergies de contact corporel pour signifier la consolation. La caresse, étant reconnue comme l'équivalence (socialement construite) de la consolation, est un geste effectué avec une énergie lente et continue, émis dans l'intention d'exprimer de la compassion ou pour transférer cet état intérieur à un autre être. On présume «naturellement » que, pour que le transfert réussisse, une certaine règle ou code social habilite les personnes à voir la manifestation de la même façon (caresse = consolation). Il s'agit ici d'enseigner un «savoir-consoler » ou un « savoirdemander de la consolation » pour permettre aux autistes de s'exprimer dans un contexte qui s'y prête et de reconnaître cet acte de réconfort, soulagement apporté à la peine de quelqu'un.

L'enseignant d'EPS a travaillé à deux reprises sur le rapport d'équivalence, caresse à consolation, avant d'aborder la situation présente. Dans les situations précédentes, le travail d'expression de la consolation avait commencé par celui des signes distinctifs de la tristesse, sans effet notable chez les adolescents; cette fois le travail est précédé par l'évocation des signes de la peur. En effet au début de la situation proprement dite, deux stagiaires présents à cette troisième séance, ont soudainement tapé avec « violence » sur la porte de la salle de danse. Cet « artifice » (à valence mésogénétique) a permis à l'enseignant de placer les trois adolescents autistes, que l'on nommera An, Jé et Ké, dans une ambiance relativement angoissante, susceptible d'engendrer de la peur.

Grâce à l'atmosphère mise en place (suite aux bruits inattendus produits par les deux stagiaires) l'enseignant s'est retiré physiquement du milieu. Il attend que celui-ci fasse rétroaction pour se placer du côté des adolescents et nommer-désigner les réactions de surprise ou de peur qu'ils sont en train de vivre. Il intervient, oralement et physiquement, alors que An, assis au sol et tellement pris par l'ambiance, le saisit par les deux bras, ce qui va l'amener à rassurer An, en le frictionnant et en lui parlant pour le rassurer :

« qui a fait du bruit? + hein? + c'était pas pour de vrai hein? + hein? + c'était pour nous faire peur ++ mais pas pour de vrai + c'est pour faire semblant + d'avoir peur + est-ce que toi tu as peur?" 
Puis l'enseignant, s'appuyant sur le milieu préalablement aménagé, conduit les trois adolescents, l'un après l'autre, à exprimer des comportements précis de consolation. Il rebondit sur la situation duelle créée avec An, pour intégrer Jé dans cette situation privilégiée visant à évoquer certains signes de consolation. On remarque ici qu'un milieu a bien été installé en amont par l'enseignant mais par la suite il fait le choix de rapidement s'en défaire en substituant Jé à son rôle de consolateur. L'enseignant suspend, intentionnellement ses interventions directes auprès des adolescents, ceci sans doute pour les rapprocher au plus près d'une réalité vécue par eux au quotidien.

\section{L'immersion des jeunes enfants en activité aquatique d'éveil}

Lactivité aquatique d'éveil (AAE) consiste avant tout en une activité de loisir en piscine où les parents viennent essentiellement pour partager avec leur enfant dans l'eau, un moment agréable et ludique. Le second objectif de cette activité familiale est lié à la motricité : permettre à l'enfant de s'adapter au milieu aquatique, d'être à l'aise dans l'eau et d'apprendre à se déplacer aussi bien en surface qu'en profondeur. La poursuite de ce second objectif est visible lors de moments ponctuels de la séance au cours desquels l'intention d'enseigner est alors manifeste. La petite fille observée (que nous nommerons $\mathrm{Az}$ ) est âgée de trois ans lors de l'extrait. Elle participe régulièrement à l'activité avec ses deux parents (que nous nommerons Paz pour le père et Maz pour la mère) depuis l'âge de six mois. C'est elle qui dirige le plus souvent la succession des jeux auxquels elle se livre dans l'eau.

Lors de la séance étudiée et dès son entrée dans le grand bassin, la petite fille se déplace entre son père et sa mère, sans matériel, en immersion, en faisant des mouvements de bras et de jambes pour avancer. Lors de son deuxième déplacement pour rejoindre $\mathrm{Paz}, \mathrm{Az}$ regarde vers le fond et aperçoit un anneau vert (d'environ $20 \mathrm{~cm}$ de diamètre). Le premier jeu alors mis en place par la fillette dans le grand bassin est d'aller chercher cet anneau. Son père aide par trois fois la petite à descendre sous l'eau pour aller chercher l'objet, dont deux fois avec succès. Puis un second jeu, centré sur un autre objet, est mis en place par l'enfant. Elle demande à son père de la projeter vers sa mère en poussant son corps par-dessous les pieds. Puis Maz fait de même en projetant Az vers $\mathrm{Paz}$. Az demande à recommencer mais Maz propose un autre jeu...

L'extrait analysé débute au moment où la mère propose à son enfant de jouer à passer à l'intérieur de grands cerceaux lestés. Son père lui explique alors comment elle doit s'y prendre et lui montre en passant lui-même à l'intérieur du cerceau immergé. La petite fille entre dans le jeu de ses parents et au bout de quelque temps en redéfinit une partie des règles. Elle passe au-dessus du cerceau, l'attrape puis passe à l'intérieur. Après avoir réitéré l'expérience huit fois, $\mathrm{Az}$ vient se reposer dans les bras de Maz. Elle regarde autour d'elle et parle à Maz (à ce moment, les paroles sont inaudibles, toutefois nous aurons confirmation, lors de l'entretien post, que $\mathrm{Az}$ demande à aller chercher l'anneau vert). Maz cherche alors avec elle en regardant vers le fond de la piscine. $\mathrm{Paz}$ arrive sous l'eau et attrape l'anneau puis le tend à Az. Il lui propose d'aller le chercher sous l'eau mais Az refuse.

Au cours de la séance la plupart des jeux mis en place sont le résultat de l'activité initiée par Az. Lépisode pris en compte débute par une première rupture dans le rythme habituel de la séance à la $2 \mathrm{e}$ minute puisque c'est Maz qui va prendre en main la mise en place d'une nouvelle situation et que cette situation va mettre en jeu un nouvel objet encore jamais utilisé par Az. En voici le récit : Maz va chercher un cerceau (dont le diamètre mesure approximativement $1 \mathrm{~m}$ ), l'immerge à une profondeur d'environ $1 \mathrm{~m} 50$ et explique à $\mathrm{Az}$ que ce cerceau est fait pour « passer dedans ». Cet objet matériel n'a jamais été utilisé précédemment par la fillette.

Paz apporte lui aussi sa part de construction à ce nouveau jeu en expliquant, par une démonstration, le « mode d'emploi » du nouvel objet. Il s'immerge et passe au travers du cerceau posé dans le fond. 


\begin{tabular}{|l|l|l|l|}
\hline 00:02 & 63 & Az & $\begin{array}{l}\text { Az est assise sur les genoux de Paz + tient Paz par le cou [ Az dit : } \\
\text { encore maman] [ Paz dit : encore maman] + [ Maz dit : Regarde ça } \\
+ \text { c'est pour aller nager dedans] Az lâche le cou de Paz + se lance } \\
\text { en avant vers Maz + avance vers Maz le visage immergé en faisant } \\
\text { des mouvements de bras et de jambes }\end{array}$ \\
\hline 64 & Maz & $\begin{array}{l}\text { Maz attrape de sa main gauche la main droite d'Az + tire Az vers } \\
\text { elle + émerge le cerceau lesté qu'elle tient dans la main droite [ } \\
\text { Maz dit }:<\ldots \text { ? }>\text { [ Paz dit : ah oui ça c'est pour passer sous l'eau + } \\
\text { c'est pour passer dessous] Maz immerge le cerceau [ Maz : <.. ?>] } \\
{[\text { Paz dit }: \text { regarde Az + regarde + comme ça] }}\end{array}$ \\
\hline 65 & Paz & $\begin{array}{l}\text { Paz s'immerge + avance sous l'eau en réalisant des bras de « nage } \\
\text { brassée »+ passe dans le cerceau + remonte près d'Az et de Maz [ } \\
\text { Paz dit : tu vois + tu passes dedans] }\end{array}$ \\
\hline
\end{tabular}

Ce jeu peut être qualifié de jeu didactique, au sens où ce qui le produit est la nécessité de progresser dans l'apprentissage du déplacement aquatique sous l'eau. Il renvoie à un enjeu de savoir précis. Nous procédons à l'analyse a priori du jeu «plongeon canard » rendu possible par la présence du grand cerceau à travers lequel le papa propose de passer. Lanalyse a priori consiste en une analyse objective et « externe » de la tâche aquatique, c'est-à-dire réalisée indépendamment de l'analyse des interactions effectives, mais rapportée aux capacités physiques d'un jeune enfant de trois ans. Pour cette analyse a priori nous proposons de voir le plongeon canard comme un problème mécanique : comment descendre au fond de l'eau contre la poussée d'Archimède et la résistance à l'avancement? Le problème principal est en effet le déplacement en profondeur sur un axe compris entre $45^{\circ}$ et $90^{\circ}$ par rapport à la surface. Ce déplacement implique d'aller contre la poussée d'Archimède, appelée force $(\mathrm{Pa})$ : $(\mathrm{Pa})$ est appliquée au centre de poussée, situé à proximité du plus grand volume corporel, c'est-à-dire le thorax. Rappelons que cette poussée est orientée verticalement vers le haut, et d'intensité égale au poids du volume d'eau déplacé par le corps immergé et qu'elle limite la résistance à l'avancement (R), composée de trois formes de résistance : la résistance frontale, la résistance de frottement et la résistance tourbillonnaire) qui se calcule selon la formule : R = KSV (K : coefficient de forme qui se déplace; $\mathrm{V}$ : vitesse de déplacement; $\mathrm{S}$ : coefficient du "maître couple », ce qui correspond à la projection de la surface du corps immergé sur un plan perpendiculaire à l'axe de déplacement).

La réussite à ce jeu dépend de l'orientation de l'axe du corps et de la puissance de propulsion des mouvements effectués avec les bras et les jambes du nageur, sachant que plus il s'éloigne de $90^{\circ}$ par rapport à la surface, plus le " maître couple » (S) augmente et par conséquent la résistance à l'avancement et plus le nageur devra fournir d'effort pour descendre. Habituellement un jeune enfant, dit « à l'aise dans l'eau », se déplace le visage immergé, le regard orienté entre 20 et $30^{\circ}$ par rapport à la surface, en apnée, en effectuant des pédalages avec les jambes et des mouvements de bras. Lorsque l'enfant a pied, la plupart du temps il se déplace librement à la verticale en sautillant ou bien à l'horizontale, visage immergé, en faisant des mouvements de jambes et de bras pour avancer. Lorsqu'il n'a pas pied il s'appuie sur le corps de ses parents ou s'aide d'objets flottants. La situation « plongeon canard» implique donc une remise en question de la motricité enfantine habituelle dans l'eau, notamment dans l'orientation du regard.

Az est une enfant que nous qualifions d'« à l'aise dans l'eau ». Tout au long des séances elle a pu développer une locomotion spécifique, de plus en plus efficace. Dans les bassins à petite profondeur, lorsqu'elle a pied, elle se déplace soit à la verticale en sautillant, soit le buste à l'horizontale avec l'angle buste - membres inférieurs légèrement fermés, visage immergé, respiration bloquée, le regard orienté à environ $20^{\circ}$ par rapport à la surface, en effectuant des mouvements de bras (type « petit chien» avec retour subaquatique et parfois des retours aériens type nage crawlée.). L'inspiration s'effectue dans l'axe du corps en relevant la tête, entraînant le plus souvent une reprise d'appuis au sol ou une longue pose en surface (le temps de plusieurs cycles respiratoires : inspiration/expiration). L'exploration de la profondeur se fait généralement dans la recherche de petits objets immergés (anneaux, coquillages algues). La descente s'effectue par les pieds. De la même manière elle se 
déplace en grande profondeur sur une distance pouvant aller jusqu'à $12 \mathrm{~m}$ (une largeur). Mais le plus souvent elle part de l'un de ses deux parents pour aller vers l'autre parent ou vers un matériel sur lequel elle peut prendre appui. Elle utilise parfois également des «frites» (long cylindre de mousse) ou encore des palmes. L'exploration de la profondeur est moins courante et s'effectue habituellement avec son père. La descente est réalisée de la même manière qu'en petite profondeur, par les pieds.

Le jeu du «plongeon canard » implique par conséquent une modification de sa motricité habituelle. Il constitue un nouveau milieu (en partie ancien, en partie antagoniste). fortement didactique (présenter une nouvelle situation et un nouveau matériel témoigne d'une intention de faire " avancer » l'apprentissage). Impulsé par Maz puis par Paz, guidant et structurant l'action de la petite fille, ce milieu va ensuite se relâcher, Maz comme Paz s'effaçant lors de la nouvelle confrontation d'Az à la situation de passage dans le cerceau immergé.

\section{Deuxième étape : l'intervenant se saisit des réponses et joue sur l'adidacticité}

C'est bien «par défaut du savoir » qu'intervient la dévolution dans les trois cas: (i) les consignes et mimes de l'entraîneur qui « taisent » la solution, devant les échecs répétés de l'athlète; (ii) la réaction de l'adolescent autiste aux « bruits » du milieu et l'incitation « douce » du professeur au travail d'expression des émotions; (iii) des régulations effectuées par les parents afin de permettre à leur petite fille d'approcher les «sensations » du plongeon canard à une nouvelle confrontation de la fillette seule face à la situation initiale. À cette étape du déroulement, aucune des situations ne permet aux apprenants d'intégrer les savoirs indispensables à l'acquisition de comportements efficaces.

Une deuxième caractéristique commune aux trois cas peut être identifiée. Les intervenants tentent de faciliter l'apprentissage en initiant un jeu de bascules topogénétiques où alternent des moments adidactiques et des moments didactiques. Durant ces moments, le milieu est réorganisé pour guider l'apprenant. Ce jeu d'alternances est déclenché par l'intervenant qui se saisit des premières réponses de l'apprenant (en situation adidactique). En voici les traits dominants actualisés dans chaque cas:

\section{L'entraînement athlétique du lancer de disque}

Lathlète, placé en exploration selon des contraintes liées aux attentes de son entraîneur, parvient à satisfaire certaines de ces attentes (position de départ, accélération du pied gauche, travail du MID), mais pas toutes. Sa motricité est dominée par son « schéma moteur » habituel dans la mesure où l'accélération de la pose du pied gauche se fait une fois que le travail du MID est terminé (phase sur un appui). Le DA débute donc lorsque l'ensemble du corps est orienté de face à la direction du lancer. Lathlète peut alors lancer avec une action habituelle des membres inférieurs: syncrétique. L'entraîneur, constatant l'échec relatif de la situation, réorganise le milieu en réduisant les possibilités d'expression de la motricité de l'athlète : il lui demande de ne pas trop écarter ses deux appuis (écart latéral, pose finale), de manière à créer une opposition sur les appuis - obligeant au respect de la chronologie souhaitable de la pose des appuis s'il veut pouvoir faire avancer sa hanche droite - et un retard plus important des épaules à leur pose.

Léchec de son ultime intervention (l'athlète ne modifie toujours pas son mode de réalisation) amène l'entraîneur à montrer la solution (action standard du MIG). Lathlète parvient alors à satisfaire l'une des attentes de l'entraîneur : en réduisant l'écart transversal, dans le sens " devant-derrière », entre les appuis, il réussit à respecter la chronologie "pose du pied gauche / travail du MID ». Toutefois, cette réduction de l'écart transversal, auquel s'annexe un redressement du corps, s'accompagne de contre effets éloignant le geste des standards de réalisation attendus par l'entraîneur et perçus par celui-ci, comme l'atteste l'entretien d'autoconfrontation de l'entraîneur : « pied gauche trop à gauche $[\ldots]$ il est aérien $(\ldots) »$. Elle court-circuite deux liaisons motrices pertinentes pour l'efficacité du lancer : d'une part, la prise d'avance en translation de l'appui gauche; d'autre part, le retard des épaules sur le bassin, ce qui diminue considérablement la mise sous tension de la chaine d'impulsion (comprenant les muscles intervenant dans la rotation des épaules par rapport au bassin). Cette modification motrice obtenue rapidement et de manière formelle entraîne par ailleurs une autre conséquence préjudi- 
ciable au lancer. La prise d'avance en torsion (rotation des épaules par rapport au bassin autour du grand axe du corps), du bassin sur les épaules, est entravée par un décalage latéral (de la droite à la gauche de l'athlète) important du pied gauche (ce qui empêche l'opposition des appuis) et une avancée de la hanche droite - occasionnée par l'action du MID - ayant lieu en même temps que celle de l'épaule droite.

Devant ces contre effets, l'entraîneur réorganise à nouveau le milieu en engageant l'athlète à éloigner la pose de l'appui gauche (augmentation de la prise d'avance en translation). Lathlète, lors des lancers suivants, fait alors varier son geste selon la façon dont il essaie de gérer les conflits qu'il rencontre. Ainsi, sur le lancer suivant, il pose l'appui gauche très à gauche - décalage latéral important - en évitant la dissociation entre les épaules et le bassin mais en dissociant le travail des appuis alors qu'ensuite, le fait d'éloigner la pose de l'appui gauche le conduit à réaliser la rotation de son pied droit et de sa ligne d'épaules avant la pose du pied gauche (pas de torsion - lancer analogue à son premier lancer). L'entraîneur, constatant l'échec de ses interventions, passe à autre chose.

Lon voit donc que le processus d'apprentissage donne lieu à différentes stratégies de resserrements partiels et variés du milieu. Ces stratégies, porteuses de degrés variables d'adidacticité, laissent plus ou moins de marge à la régulation par l'athlète de sa motricité. Les situations ainsi créées sont elles aussi porteuses d'échec à court terme.

\section{L'éducation des autistes par la danse}

Le tapage terminé, l'enseignant s'agenouille près de An qui sourit et qui lui saisit les deux bras pour les emmener vers lui. L'enseignant pose sa main droite sur le torse de An et sa main gauche sur son dos avant de le frotter doucement. L'enseignant balance, berce An tout en lui parlant.

C'est l'adolescent (An) qui crée une situation à valence affective en amenant l'enseignant sur son territoire - le milieu à ce moment peut être défini comme "lâche ». C'est l'enseignant qui transforme, avec ingéniosité, cette relation affective en situation de travail profitable aux autres adolescents, en refermant le milieu, élevant ainsi la situation proposée par l'ado- lescent en milieu pour apprendre pour tous. Il incite Jé à consoler An, à entrer en contact avec An :

« tu le prends? + prends-le comme moi ++ prends- le dans tes bras comme ça voilà + et tu le consoles ++ comme ça »

L'enseignant montre à Jé comment tenir An, quels sont les gestes à réaliser (frotter doucement le dos...), puis petit à petit personnalise la consolation de An et enfin y intègre le contexte maternel lequel peut être également évoqué directement par Jé :

" et peut être que... + qu'An quand il est malheureux + quand il a eu peur ++ et ben sa maman le console comme ça aussi »

La réaction de An ne se fait pas attendre, il saisit les mains de Jé et les place sur son ventre, amenant Jé à le serrer plus fort entre ses bras. L'enseignant continue à impulser des nouvelles actions, par exemple regarder la personne que l'on console. Le milieu se remplit alors de nouveaux objets corporels sollicitant l'adaptation des adolescents l'un à l'autre.

L'enseignant ici a répondu à la demande d'An d'être rassuré et a intégré Jé à cette demande (la substitution de l'enseignant par Jé détermine une bascule topogénétique) d'où une implication plus grande de la part de Jé et de An et une accélération conséquente du temps didactique. Il apparaît à ce moment encore plus décisif que l'enseignant ait préparé en amont l'atmosphère mésogénétique (environnement musical, bruitages, paroles...) pour être assuré de rebondir sur une réaction, une demande affective de la part d'un ou de plusieurs adolescents. Dans cet extrait la réussite de la dévolution est visible lorsque Jé, initie un nouveau geste qui est de caresser la tête de An. Cette action avec un semblable (décidée et produite par Jé seul) est à l'évidence un produit de la dévolution.

Dans cette séquence, c'est bien l'initiative de Jé (caresser la tête de An avec sa main gauche puis le haut du dos de An, ce qui provoque chez An des sourires) qui permet à l'enseignant d'entamer le moment dévolutif. On pourrait croire que Jé, compte tenu du fait que l'enseignant a déjà travaillé antérieurement avec ces adolescents sur la consolation, sur les postures et gestes à réaliser (dans d'autres séances ou situations d'enseignement), a intégré ce que veut l'enseignant; et dans ce cas Jé serait sensible aux attentes de l'enseignant. Mais si l'on sait que Jé (et cet adolescent en particulier) rencontre de sérieux problèmes de prise 
d'initiative, qu'il attend toujours l'accord de l'enseignant et que cet accord soit institué par le regard ou la parole pour entreprendre une tâche, alors on peut penser à une dévolution réussie. Jé est sujet également à de tenaces difficultés d'attention portée à ce qu'il réalise, de sorte qu'en quelques secondes Jé peut se désintéresser totalement de ce qu'il fait. Il paraît clair que dans cette séquence Jé a pris la responsabilité de la tâche qu'il réalise : le geste réalisé n'est en rien une reproduction de ce que vient de faire l'enseignant. Jé est bien initiateur, créateur de ce geste à ce moment précis. Il est vraisemblable que l'implication didactique de Jé est dépendante également des situations organiquement coopératives instaurées par le professeur, qui a érigé le comportement de An qui, à cet instant, est demandeur de contact, en « objet du milieu » propre à la communication.

\section{L'immersion des jeunes enfants en activité aquatique d'éveil}

Lors de sa première tentative de passage dans le cerceau au fond de l'eau, Az se déplace horizontalement et passe au-dessus du cerceau. Lors d'une seconde tentative, Az s'immerge tout en se tenant à Maz et attrape le cerceau. Maz, qui tient Az dans les bras lui parle et reprend le cerceau qu'elle met dans le fond. Az émerge alors sans être passée dans le cerceau. Paz propose alors de mettre le cerceau plus haut (le haut du cerceau touche la surface). Az se déplace horizontalement en immersion en faisant des mouvements de bras et de jambes, attrape le cerceau avec les deux mains, baisse la tête et passe au travers du cerceau.

\begin{tabular}{|l|l|l|l|}
\hline 2'00 & 87 & {$[\mathrm{M}=\mathrm{Az}]$} & $\begin{array}{l}\text { Az s'assoie sur les genoux de Maz + se tient au cou de Maz [ } \\
\text { Maz dit }:<\ldots ?>]\end{array}$ \\
\hline 88 & $\begin{array}{l}{[\quad \mathrm{M}} \\
=\mathrm{Paz}]\end{array}$ & $\begin{array}{l}{[\text { Paz dit }: \text { regarde on le met là regarde] Paz remonte le cerceau }} \\
\text { jusqu'à ce que le haut du cerceau touche la surface [ Maz dit : } \\
\text { on le met là + ça te va ?] [ Paz dit : allez + dans le cercle] [ Maz } \\
\text { dit : dans le cercle] }\end{array}$ \\
\hline & 89 & {$[\mathrm{M}=\mathrm{Az}]$} & $\begin{array}{l}\text { Az replace son masque + se lance en avant + attrape le cerceau } \\
\text { à deux mains + incline la tête + passe dans le cerceau en } \\
\text { battant des jambes + attrape le cou de Paz [ Paz rit ] }\end{array}$ \\
\hline
\end{tabular}

Maz présente le cerceau en surface et tend sa main droite à Az sous l'eau au travers du cerceau. Az se déplace horizontalement vers le cerceau et attrape la main de Maz pour passer un peu plus en profondeur dans le cerceau. Puis, après ces diverses procédés mis en œuvre pour inciter $\mathrm{Az}$ à passer dans le cerceau immergé, nous constatons une rupture marquée par le fait qu'Az se retrouve de nouveau « seule » face à la situation initiale. Le cerceau est replacé au fond et Paz demande à Az cette fois-ci de plonger tout au fond. Az se lance pour passer seule dans le cerceau qui n'est tenu ni par Maz ni par Paz. Elle se déplace à la surface, horizontalement en battant des jambes et des bras. Arrivée audessus du cerceau, Az se penche, attrape le cerceau, le monte à sa hauteur et passe à l'intérieur sans s'immerger davantage. Ses parents rient de cette initiative détournée.

\begin{tabular}{|c|c|c|c|}
\hline \multirow[t]{6}{*}{$00: 03$} & 13 & Maz & Maz prend Az dans ses bras [ Maz dit: super] \\
\hline & 14 & $\mathrm{Az}$ & $\begin{array}{l}\text { Az s'assoie sur les genoux de Maz + passe son bras gauche } \\
\text { autour du cou de Maz + se tourne vers Paz }\end{array}$ \\
\hline & 15 & $\mathrm{Paz}$ & $\begin{array}{l}\text { [ Paz dit : attends attends] Paz prend le cerceau [ Paz dit : on } \\
\text { va réussir à plonger encore plus en dessous + regarde] + Paz } \\
\text { pose le cerceau au fond de la piscine entre Maz et lui }\end{array}$ \\
\hline & 16 & $\mathrm{Az}$ & $\begin{array}{l}\text { Az regarde Paz }+ \text { se met debout sur le genou droit de Maz } \\
\text { [ Paz dit : pour plonger là }+<\ldots \text { ? }>\text { ] Az se jette en avant }+ \\
\text { se déplace à l'horizontal }+ \text { le regard à } 40^{\circ} \text { par rapport à } \\
\text { l'horizontale }+ \text { fait des mouvements de jambes }+ \text { tend les } \\
\text { bras vers le cerceau }+ \text { attrape le cerceau }+ \text { passe dans le } \\
\text { cerceau }\end{array}$ \\
\hline & 17 & $\mathrm{Paz}$ & $\begin{array}{l}\text { Tend la main gauche au travers du cerceau + attrape le } \\
\text { cerceau de la main droite + tire Az vers lui de la main } \\
\text { gauche }\end{array}$ \\
\hline & 18 & $\mathrm{Az}$ & $\begin{array}{l}\text { Az émerge la tête + attrape le cou de Paz [ Paz rit et dit : éh } \\
\text { éh éh + elle attrape le machin] [ Maz rit et dit: si je ne vais } \\
\text { pas au cerceau le cerceau viendra à moi :] }\end{array}$ \\
\hline
\end{tabular}


Enfin, Maz propose à nouveau de passer dans le cerceau, en le tenant plus près de la surface et en tendant la main sous l'eau. Az se déplace horizontalement en battant des jambes, le visage immergé et saisit la main de Maz, passe dans le cerceau, lâche la main de Maz pour enfin émerger. Cette situation se répète encore trois fois avant qu'Az se décide à changer de jeu.

Cette série de comportements peut être interprétée de la manière suivante :

Durant la première partie de la séance, en grand bassin, Az prend la responsabilité majeure de la construction de situation de jeu. Mais les thèmes abordés n'engagent pas ou peu de modifications particulières de sa locomotion aquatique (aquacité). À la deuxième minute, nous constatons la première "bascule topogénétique». Celle-ci se traduit par l'introduction d'un nouvel objet matériel, potentiellement objet d'apprentissage. Maz use de techniques mésogénétiques pour pointer du doigt une «ignorance » au sens de Joshua et Félix (2002), dans le milieu alors stable dans lequel évolue $\mathrm{Az}$.

Ainsi, dans un premier temps les parents d'Az définissent une situation porteuse de savoir nouveau. Le milieu alors très "lâche » est ensuite refermé pour ne laisser que très peu de liberté à l'enfant dans ses choix. Lintention "d'enseigner » apparaît alors visible pour l'enfant. Cette rupture marque également le passage de situations non didactiques à une situation à caractère didactique.

Par ailleurs, le fait que l'enfant produise la bonne réponse indique nécessairement une modification de sa motricité habituelle. La part d'intervention des parents se présente donc comme chronogène (relative à l'avancée des apprentissages). On peut supposer qu'à travers cette intervention, l'enfant ressente une certaine pression temporelle (chronogénètique). Ainsi, sur cette courte durée, elle est censée apprendre à : répondre à la demande de ses parents en construisant une nouvelle solution, face à ce qui lui échappe (passer sous l'eau dans le cerceau).

Toutefois, les premières réponses produites par $\mathrm{Az}$ ne lui ont pas permis d'atteindre l'objectif fixé par ses parents. Par conséquent Paz a décidé de modifier la situation. Son action de régulation semble retirer une grande partie du sens de la situation. Cette modification permet d'une part, de ne pas tenir l'enfant en échec, et d'autre part, de "forcer » tout de même la fillette à un changement dans sa motricité habituelle : pour se déplacer en s'enfonçant dans l'eau, elle accepte une légère flexion de la tête et une modification très fine de l'axe vertébral. L'enfant par cet essai d'accommodation motrice répond par conséquent en partie aux attentes de Paz et Maz.

Lors de cette situation que nous qualifions de fortement didactique nous notons l'apparition d'une rupture marquée par le fait que l'enfant, jusque-là guidée, est confrontée « seule » à la situation initiale. La responsabilité de l'apprentissage demeure alors à sa seule charge sur une durée de quelques minutes (moment de dévolution).

Apparaît donc une nouvelle bascule topogénétique, mais cette fois, impulsée parMaz et Paz, au sein d'une situation que nous qualifions de didactique, car elle met en jeu les savoirs intentionnellement choisis par les parents. Le milieu, précédemment très « guidant » s'ouvre encore pour laisser un éventail plus large de réponses possibles. C'est par conséquent de nouveau par le biais de techniques mésogénétiques que s'effectue cette nouvelle territorialisation de l'espace décisionnel. Ainsi l'enfant s'engage par l'action dans la série de problèmes présents dans la situation. Elle se doit de trouver les moyens de répondre pour satisfaire aux attentes de ses parents. L'enfant se trouve face à un creux de savoir qu'elle va devoir combler.

Nous devons également considérer le décalage entre les potentialités de l'enfant et ce qu'on lui demande de faire. Les exigences de la situation sont sans doute ici trop complexes pour espérer obtenir la réponse voulue. En conséquence, Az redéfinit les règles du jeu en descendant légèrement pour réussir à attraper le haut du cerceau et passer dedans. Une partie de la demande étant réalisée, Az peut alors passer à un autre jeu.

\section{Troisième étape : l'institutionnalisation des réponses motrices}

Nous avons montré que la dévolution se présente sous la forme d'un processus inscrit dans un jeu d'al- 
ternances de moments didactiques et adidactiques. L'institutionnalisation des réponses adéquates ne peut donc être circonscrite à une situation adidactique isolée. En outre, les cas étudiés montrent un décalage temporel variable entre l'émergence des éléments d'adidacticité et l'apparition des éléments d'institutionnalisation.

\section{L'entraînement athlétique du lancer de disque}

C'est en fin de cycle d'entraînement que l'athlète, au gré des répétitions et par l'adjonction de composantes facilitatrices en amont (entrée en volte), réussit à utiliser efficacement son MIG suite à la reprise de l'appui droit. Il parvient alors de lui-même à tirer profit de l'utilisation d'une action du MIG analogue à celle attendue par l'entraîneur comme moyen d'optimiser sa performance. Le moment dévolutif a permis finalement d'engager l'apprentissage de l'athlète sur du long terme, dans un processus de résolution des problèmes liés à l'utilisation du MIG. Ses effets sont donc différés. L'institutionnalisation du geste technique efficace a eu lieu en fin de cycle (9e séance), suite à une succession de situations didactiques abordant parmi d'autres thèmes celui du retour du MIG au sol :

« voilà/reste dessus/reste dessus//ça c'est bien/tu vois eh/ ça va plus loin tu t'énerves pas/mais ouais/c'est comme ça qu'il faut que tu lances tout le temps/d'accord? "

De nombreux feedbacks positifs ont jalonné les interventions de l'entraîneur entre la deuxième et la neuvième séance, souvent dénués d'allusion à un contenu technique ( "c'est bien », " oui »...) et faisant office de transition pour aborder un autre point technique. Seul ce passage révèle une satisfaction globale de l'entraîneur : il fait suite à des situations didactiques prenant pour thème le retour du MIG, et cesse de donner lieu à l'introduction d'un nouvel objet de savoir. En outre, cet épisode est apparu décisif sur le plan de l'évolution de la motricité de l'athlète (le nombre de lancers conformes aux attentes de l'entraîneur concernant le retour du MIG est en nette augmentation suite à cette intervention). Voilà pourquoi nous l'avons retenu comme moment essentiel d'institutionnalisation du savoir technique étudié. Lobservation est donc essentielle parce qu'elle montre que rencontrer son ignorance n'est pas la condi- tion pour apprendre, mais celle pour commencer à étudier. C'est donc bien le moment où la dévolution peut advenir (ce qui se voit au fait que l'étude s'engage), et la régulation réussir (ce qui se voir au fait que l'étude est dirigée).

\section{L'éducation des autistes par la danse}

Ce cas présente une plus grande unité temporelle car les moments de dévolution et d'institutionnalisation semblent coincider. Pour clore le moment de dévolution, l'enseignant referme le milieu et cesse ses interventions, en institutionnalisant le geste de Jé - la caresse - comme étant l'une des expressions de la consolation. Il poursuit cette institutionnalisation en refusant d'établir une équivalence entre les chatouilles que réclame An (mouvements rapides, énergiques), et' l'expression de la consolation :

\footnotetext{
«oui... tu peux lui toucher la tête comme ça pour le consoler + c'est comme ça que font les mamans et les papas quand ils consolent leur enfant ++ hein ? + ouais... c'est super ça ++ on a besoin + c'est normal hein ? + hein ? + on fait comme ça + et puis là y a le sourire " "Hein + c'est pas des guilis hein? + on fait pas des guilis là + c'est consoler + c'est pas pareil ++ hein? + là on console +++ An ».
}

Enfin avant de « relâcher » le milieu pour Jé et An, l'enseignant propose à An de prendre en charge la consolation en s'occupant de Jé :

\footnotetext{
« tu peux consoler aussi + tu peux prendre pareil ++ viens + tu peux prendre aussi Jé + prends Jé par le cou + prends Jé aussi »
}

An, suite à la demande de l'enseignant, finit par poser et frotter sa tête sur le bras gauche de Jé qui reprend son rôle de consolateur et le prolonge. L'enseignant, laissant le milieu « lâche » pour Jé et An, en profite pour reprendre une phase de démonstration avec Ké.

\section{L'immersion des jeunes enfants en activité aquatique d'éveil}

Leevénement étudié lors de la séance choisie présente les traits d'une institutionnalisation différée. En effet nous observons lors de cette séance des encouragements et des «validations " des certains gestes ou actions : 
"Paz dit: ouais super!"

«Maz: ouais (rire) c'est bien (rire)»

Après plusieurs séances mettant en jeu le plongeon canard, dans des situations variées (avec palmes, en petite profondeur, en plongeant d'un tapis), nous constatons (plus d'un an après) la réalisation d'un « vrai » plongeon canard, lequel évoluera très rapidement au cours de la même séance. C'est lors de cette séance notamment que le plongeon canard est institutionnalisé par Paz : «Paz dit : tu fais un plongeon canard » ce qui sous entend que cet élément fait partie du registre des possibles d'Az et a été naturalisé comme tel. Paz continue tout de même à guider $\mathrm{Az}$ afin d'améliorer encore la technique de bascule des fesses au-dessus de la tête : Paz dit: "faut bien lever les fesses avant...il faut que tu nages et que tu sautes à l'extérieur comme si tu plongeais »). Et chaque progrès est encore validé au fur et à mesure de la séance. Un rapprochement peut être établi avec la démarche de l'entraîneur en athlétisme : la direction sur un long terme, supposant une certaine organisation de la progression par l'intervenant

\section{Conclusion}

Le travail présenté s'est attaché à travailler l'un des concepts fondateurs de la didactique - le concept de dévolution - dans un cadre particulier (non scolaire, pratique d'APSA) et selon une perspective comparatiste. La relation didactique dans le domaine sportif révèle une pluralité de types de transmission possibles, présentes dans les domaines d'intervention choisis (athlétisme de haut niveau, danse en institut spécialisé avec adolescents autistes, activités aquatiques avec de jeunes enfants). Bien que conscients de l'impact de différents facteurs (tels que les représentations des acteurs ou la charge affective de la relation) dans l'émergence de cette pluralité (notamment à travers leur poids sur les formes prises par les transactions), nous avons centré notre étude sur la comparaison de ces formes, dans les trois cas abordés, afin de contribuer à l'approfondissement du concept de dévolution.

Les trois étapes décrites permettent de rendre compte des particularités de la dévolution dans des APSA non scolaires. En tant que processus évolutif, elle va de pair avec les modifications du milieu dans lesquelles elle s'inscrit.
L'orientation anthropologique de cette étude a permis de montrer que les descriptions théoriques « naives » de la dévolution issues de l'approche de la relation maître/élève en classe doivent être remaniées pour rendre compte des pratiques d'APSA non scolaires. La distribution chronogénétique des objets de savoir, caractéristique des curricula scolaires soumis à de fortes pressions institutionnelles, est ici plus éclatée et plus complexe. Lon se situe également à distance des pratiques d'ingénierie didactique qui conçoivent et organisent des principes de construction de situations adidactiques propres à faire émerger la dévolution. Nous avons au contraire montré comment les pratiques dévolutives et leurs effets peuvent s'inscrire dans une succession particulière d'événements et de situations qui caractérisent notre domaine d'étude.

Les régularités constatées nous ont permis de rendre compte d'habitudes d'intervention «naturelle » intégrant, comme par une nécessité fonctionnelle, des éléments dévolutifs. Nous avons ainsi pu dégager un modèle qui rend compte de ces pratiques en montrant leur lien avec l'épistémologie et l'apprêt didactique des intervenants.

Au-delà des questions fondamentales évoquées précédemment, il faut souligner deux aspects qui peuvent expliquer en partie ces particularités et difficultés de transmission. Il est coutumier, dans les représentations ordinaires, de considérer qu'en activités physiques et sportives le fait de "savoir faire » donne immédiatement compétence à "savoir enseigner » les pratiques que l'on maîtrise. Dès lors, les apprentissages apparaissent surtout comme l'acquisition de formes et de sensations que l'intervenant perçoit comme essentielles. Cette façon d'aborder les questions de diffusion des savoirs conduit invariablement à des présentations saturées en consignes didactiques, en ostensions gestuelles ou en guidages sensoriels divers en cas d'échec.

Or il est apparu dans les cas étudiés que ce type d'impasse constitue la condition essentielle de l'intervention d'un «jeu dévolutif »: la mise en jeu de micro-épisodes dévolutifs favorise, par bascules topogénétiques successives, la mise en œuvre d'une dévolution « incidente " plus globale, identifiable par ses effets à moyen (séance) ou long terme (cycle). C'est cette dernière qui permet alors à l'apprenant 
de dépasser l'obstacle qui entravait sa progression jusque-là.

Une seconde dimension doit être évoquée pour comprendre les limites que rencontrent intervenants, dans la construction de contenus adaptés à transmettre. Les savoirs en activités physiques et sportives sont multiples, divers et peu structurés. Ces savoirs sont issus des pratiques. Dès lors que le primat est donné à la pratique, le pragmatisme fait souvent loi. Cette attitude conduit à considérer que, hors de l'expérience, il n'y a point de salut. C'est le fait de faire qui assure toute compétence dans la détermination des savoirs utiles et dans leur diffusion. Il apparaît donc que la nature du rapport qu'entretiennent les intervenants avec la pratique considérée, prévaut sur la nature des savoirs, dans l'évolution des transactions didactiques.

Ce travail ouvre des perspectives d'approfondissement de la démarche comparatiste entreprise. Les méthodes utilisées pourront être adaptées à l'étude comparée d'autres phénomènes didactiques.

\section{NOTES}

1. Le préfixe «trans » vise à souligner que toute transmission de savoir s'accomplit à travers un milieu « entre » le professeur et les élèves où chacun accomplit une part d'action.

\section{BIBLIOGRAPHIE}

Bachelard, G. (1938/1997). La formation de l'esprit scientifique. Paris : Vrin.

Brousseau, G. (1998). Théorie des situations didactiques. Grenoble : La Pensée Sauvage.

Brousseau, G. (1986). Fondements et méthodes de la didactique des mathématiques. Recherches en didactique des mathématiques, 7/2, 33-116. Grenoble: La Pensée Sauvage.

Brousseau, G. (1982). Les objets de la didactique des mathématiques. Ingénierie didactique. Deuxième école d'été de didactique des mathématiques, Olivet (texte non publié).

Brousseau, G., Centeno, J. (1991). Rôle de la mémoire didactique de l'enseignant. Recherches en didactique des mathématiques, 11/2.3, 167-210. Grenoble: La Pensée Sauvage.

Chevallard, Y. (1992). Concepts fondamentaux de la didactique : perspectives apportées par une approche anthropologique. Recherches en didactique des mathématiques, 12/1, 73-111. Grenoble: La Pensée Sauvage.

Chevallard, Y. (1989). Le concept de rapport au savoir. Rapport personnel, rapport institutionnel, rapport officiel. Séminaire de Didactique des Mathématiques et de l'Informatique, 26 juin, Université Joseph Fournier, Grenoble I. Document interne ${ }^{\circ} 108$.

Chevallard, Y. (2002). Organiser l'étude - 3. Ecologie et régulation. In J.-L. Dorier, M. Artaud, M. Artigue, R.

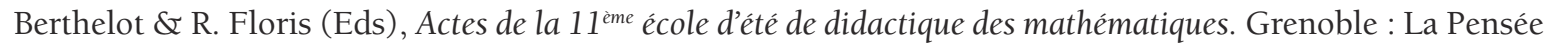
Sauvage.

Comiti, C., Grenier, D., \& Margolinas C. (1995). Niveaux de connaissances en jeu lors d'interactions en situation de classes et modélisation de phénomènes didactiques. In G. Arsac et al. (Eds.), Différents types de savoirs et leur articulation (pp. 92-113). Grenoble: La Pensée Sauvage.

Frith, U. (1992). L'énigme de l'autisme. Paris : Odile Jacob.

Guillerme, J. (1985). Technologie. Paris : Encyclopaedia Universalis.

Joshua, S., Félix, C. (2002). Le travail des élèves à la maison : une analyse didactique en termes de milieu pour l'étude. Revue Française de Pédagogie, 141, 89-97. Paris : INRP.

Laban, R. (2003). La danse moderne éducative. Bruxelles : Editions Complexe et Centre national de la danse.

Loquet, M., Roncin, E., \& Roessle, S. (2007). Laction conjointe dans le système didactique en activités physiques, sportives et artistiques : les formes non verbales de communications didactiques. In G. Sensevy \& 
A. Mercier (Eds.), Agir Ensemble. L'action didactique conjointe du professeur et des élèves (pp. 123-151). Rennes: PUR.

Marsenach, J. (1991). Education Physique et Sportive : quel enseignement? Paris : INRP.

Perrin-Glorian, M.-J., Hersant, M. (2003), Milieu et contrat didactique, outils pour l'analyse de séquences ordinaires. Recherches en didactique des mathématiques, 23/2, 217-276. Grenoble: La Pensée Sauvage.

Perrin-Glorian, M.-J. (1999). Problèmes d'articulation de cadres théoriques : l'exemple du concept de milieu. Recherches en Didactique des Mathématiques, 19/3, 279-322. Grenoble : La Pensée Sauvage.

Schubauer-Leoni, M.-L., Leutenegger, F, Ligozat, F, \& Fluckiger, A. (2007). Un modèle de l'action conjointe professeur-élèves : les phénomènes didactiques qu'il peut/doit traiter. In G. Sensevy \& A. Mercier (Eds.), Agir Ensemble. L'action didactique conjointe du professeur et des élèves. Rennes : PUR.

Sensevy, G. (2002). Des catégories pour l'analyse comparée de l'action du professeur : un essai de mise à l'épreuve. In P. Venturini, C. Amade-Escot \& A. Terrisse (Eds), Etude des Pratiques Effectives : l'approche des didactiques (pp. 25-47). Grenoble : La Pensée Sauvage.

Sensevy, G. (2007). Des catégories pour décrire et comprendre l'action didactique. In G. Sensevy \& A. Mercier, (Eds.), Agir Ensemble. L'action didactique conjointe du professeur et des élèves. Rennes: PUR.

Veyne, P. (1978). Comment on écrit l'histoire. Paris : Seuil.

Vivès, J., Vigarello, G. (1983). Technique corporelle et discours technique. Education Physique et Sportive, 184, 40-47. Paris : Revue EPS.

Vivès, J., Vigarello, G. (1986). Discours de l'entraîneur et technique corporelle. Education Physique et Sportive, 200-201, 146-152. Paris : Revue EPS.

Vivès J., Vigarello, G. (1989). Gestualité de l'entraîneur et technique corporelle. Education Physique et Sportive, 216, 12-18. Paris : Revue EPS.

Vygotski, L.S. (1934/1997). Pensée et langage - La dispute. Paris : SNEDIT 